\title{
A importância da partilha da informação clínica: o relato de caso de uma reação adversa
}

\author{
Alexandra Cadete, ${ }^{*}$ Lara Sutil,* Carine Silva, ${ }^{*}$ Joana Simões*
}

\begin{abstract}
RESUMO
Introdução: Ainda que pouco consensual, a terapêutica antiemética é amplamente utilizada na prática clínica com o objetivo de minimizar a desidratação e diminuir a necessidade de fluidoterapia endovenosa. A escolha do antiemético é de extrema importância, dada a incidência de reações adversas que pode alcançar os $25 \%$ em idade pediátrica.

Descrição do caso: Adolescente do sexo masculino, com 12 anos de idade, recorreu ao serviço de urgência de um hospital privado por surgimento no próprio dia de cefaleias, um pico febril e um episódio de vómito. Foram administrados paracetamol e metoclopramida endovenosos com melhoria das queixas. Contudo, algum tempo depois, reiniciou um quadro de cefaleias frontais intensas e agitação psicomotora, que motivou a transferência para o serviço de urgência do Serviço Nacional de Saúde. À admissão apresentava-se muito ansioso, queixoso, agitado e desconfortável. No início da observação iniciou um conjunto de movimentos anómalos que dificultavam a comunicação e a realização do exame objetivo, mas sem nunca perder a consciência. Suspeitou-se de uma reação extrapiramidal à administração de metoclopramida endovenosa, tendo sido administrado biperideno endovenoso, com reversão total do quadro clínico. Comentário: Apesar de pouco frequentes, as reações distónicas agudas podem surgir com a dose terapêutica de metoclopramida. Os médicos de medicina geral e familiar contactam com utentes de todas as idades e, como tal, relembra-se a importância de ter sempre presente os possíveis efeitos adversos da medicação, assim como do registo e transmissão da informação relativa às atitudes terapêuticas realizadas, que neste caso foram essenciais. Em idade pediátrica, como alternativa à metoclopramida, a administração de fármacos com segurança bem estabelecida, como o ondansetron e a domperidona, é aconselhada.
\end{abstract}

Palavras-chave: Extrapiramidal; Metoclopramida; Pediatria; Antieméticos.

\section{INTRODUÇÃO}

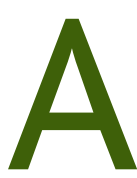

inda que pouco consensual, a terapêutica antiemética é amplamente utilizada na prática clínica com o objetivo de minimizar a desidratação e diminuir a necessidade de fluidoterapia endovenosa. ${ }^{1}$ De acordo com o seu local de ação, estes fármacos podem ser antagonistas dos recetores de dopamina, histamina ou serotonina (Quadro I). ${ }^{2}$

Os antieméticos mais usados são os antidopaminérgicos que agem através do bloqueio dos recetores de dopamina do trato gastrointestinal e do sistema nervoso central. O bloqueio destes recetores a nível do trato gastrointestinal permite estimular a motilidade gastrointestinal, aumentar o tónus do esfíncter esofágico inferior, inibir a produção de secreções e diminuir o fluxo sanguíneo para as mucosas. O bloqueio ao nível do sistema nervoso central permite obter o efeito desejado, que é a inibição do vómito, mas está mais frequentemente associado a efeitos

* USF Águeda + Saúde adversos de libertação extrapiramidal, como as crises distónicas, que podem surgir nas primeiras 24 a 48 horas após a toma, dado que a atividade motora é regulada pelo sistema extrapiramidal através dos neurotransmissores excitatórios colinérgicos e inibitórios dopaminérgicos; logo, o bloqueio dos últimos tem um efeito excitatório com consequente aumento da atividade motora de uma forma descontrolada. ${ }^{2}$ As crises distónicas consistem em contrações musculares que conduzem ao aparecimento de movimentos repetitivos de torção, espasmos ou posturas anormais e cuja tradução clínica depende do grupo muscular afetado (Quadro II). ${ }^{2-3}$

A incidência de reações extrapiramidais após a administração de metoclopramida é de $25 \%$ em idade pediátrica e idosos versus 0,5 a $1 \%$ nos adultos. ${ }^{3-4}$ Pensa-se que a maior permeabilidade da barreira hematoencefálica das crianças e idosos, com consequente facilidade para a penetração dos fármacos no sistema nervoso central, é uma das razões pelas quais essas faixas etárias são mais suscetíveis de sofrer uma reação extrapiramidal. ${ }^{2}$ 
Perante uma clínica relativamente aparatosa e na ausência de suspeita de reação extrapiramidal, o diagnóstico diferencial deve ser sempre feito com outras patologias neurológicas ou que causem alterações neurológicas, como: convulsão, crise conversiva, alterações eletrolíticas, lesão das cordas vocais, hiperventilação, tétano, entre outras. ${ }^{2-3}$

Relativamente ao tratamento podem ser usadas várias opções como anticolinérgicos ou benzodiazepinas. Os primeiros, biperideno ou difenidramina, restauram o equilíbrio entre agonistas e antagonistas da atividade muscular com resolução rápida do quadro. As benzodiazepinas não interferem no equilíbrio dos neurotransmissores, atuando através da indução de um relaxamento muscular e da sedação com alívio dos sintomas enquanto o fármaco é metabolizado. ${ }^{2}$

O caso que se segue descreve uma reação extrapiramidal num adolescente, onde a partilha da informação clínica entre os dois médicos, de instituições diferentes, que estiveram envolvidos foi fundamental para a rápida e bem-sucedida resolução do episódio de urgência.

\section{DESCRIÇÃO DO CASO}

Relatamos o caso de um adolescente do sexo masculino, 12 anos de idade, caucasiano, primeiro filho de uma fratria de dois. Como antecedentes pessoais de relevo salientava-se uma gastroenterite a Salmonella com choque hipovolémico aos seis anos de idade; sem alergias medicamentosas conhecidas e com o Programa Nacional de Vacinação atualizado, sem vacinas extraprograma. Como antecedentes familiares de relevo registou-se uma hipertensão arterial paterna.

Ao início da tarde do dia 13 de junho de 2014 recorreu ao serviço de urgência de um hospital privado, por apare-

\section{QUADRO I. Fármacos antieméticos e seus locais de ação}

\begin{tabular}{l|l} 
Antiemético & Local e mecanismo de ação \\
\hline Metoclopramida, domperidona & Antagonista do recetor de dopamina \\
\hline Dimenidrinato & Antagonista do recetor de histamina \\
\hline Ondansetron & Antagonista do recetor de serotonina
\end{tabular}

cimento no próprio dia de um quadro de cefaleia frontal intensa associada a um pico febril de $38,3{ }^{\circ} \mathrm{C}$ e a um vómito alimentar pós prandial. Foram administrados $500 \mathrm{mg}$ de paracetamol e $5 \mathrm{mg}$ de metoclopramida endovenosos com melhoria das queixas. Contudo, enquanto ainda permanecia em observação, reapareceu um quadro de cefaleias frontais intensas associadas a agitação psicomotora, que motivou a transferência para o serviço de urgência do Serviço Nacional de Saúde para esclarecimento do quadro clínico.

À admissão, o adolescente apresentava-se muito ansioso, queixoso, agitado, desconfortável, com frequência cardíaca de 86 bpm, pressão arterial de 125/68mmHg e temperatura axilar de $36,9^{\circ} \mathrm{C}$. No início da observação iniciou movimentos de flexão e extensão da língua e dos membros superiores que dificultavam a comunicação e a realização do próprio exame objetivo. O restante exame físico não apresentava alterações, nomeadamente exantemas, petéquias ou sinais meníngeos. Perante estes achados, posteriores à administração de metoclopramida endovenosa, suspeitou-se de imediato de um quadro de síndroma extrapiramidal, pelo que foi administrado $2,5 \mathrm{mg}$ de biperideno endovenoso numa hora, com reversão do quadro. Analiticamente, de salientar uma leucocitose de

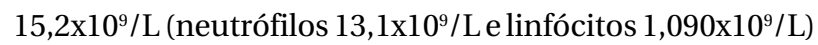
e proteína $\mathrm{C}$ reativa de $6,28 \mathrm{mg} / \mathrm{dL}$. Por persistência de

\section{QUADRO II. Manifestações clínicas de uma reação extrapiramidal2-3}

\begin{tabular}{l|l} 
Músculo/grupo muscular afetado & Clínica/Manifestação \\
\hline Palpebral & Blefaroespasmo (encerramento palpebral) \\
\hline Facial, laríngea, mandibular, língua & $\begin{array}{l}\text { Crises bucolinguais (movimentos de deglutição ou mastigação repetitivos, engasgos, protusão da } \\
\text { língua, assimetria facial) }\end{array}$ \\
\hline Ocular & Crises oculógiras (desvio conjugado do olhar) \\
\hline Laríngea e faríngea & Distonia (disfonia, estridor, disfagia) \\
\hline Cervical & Torcícolo (contratura cervical mantida ou espasmódica, desvio lateral da cabeça) \\
\hline Paravertebral & Opistótono (hiperextensão do tronco) \\
\hline Membros inferiores & Movimentos repetidos de extensão e flexão \\
\hline
\end{tabular}


febre e cefaleias frontais intensas durante a permanência no serviço de urgência foi internado para vigilância e consolidação terapêutica. Teve alta, três dias depois, com o diagnóstico de síndroma virusal, complicado de síndroma extrapiramidal após administração de metoclopramida. Ficou escrito no processo clínico do utente, bem como no seu Boletim de Saúde Infanto-Juvenil, esta reação adversa. Foi aconselhado aos pais e ao adolescente que referissem este episódio em observações médicas futuras.

\section{COMENTÁRIO}

Apesar de pouco frequentes em idade pediátrica, as reações distónicas agudas podem surgir mesmo com a dose terapêutica de antieméticos, mais frequentemente com a metoclopramida.

Pelo facto de os médicos de medicina geral e familiar contactarem com utentes de todas as idades relembra-se a importância de ter sempre presente os possíveis efeitos adversos da medicação, assim como do registo e partilha de informação clínica, que neste caso foi essencial.

$\mathrm{O}$ uso de fármacos antieméticos em idade pediátrica tem o objetivo de diminuir a necessidade de fluidoterapia endovenosa e até mesmo um internamento. Como alternativa à metoclopramida, a administração de fármacos com segurança bem estabelecida, como o ondansetron e a domperidona (apesar de antidopaminérgico, atravessa a barreira hemato-encefálica em reduzidas quantidades, ao contrário da metoclopramida), é aconselhada. ${ }^{1,5}$

\section{REFERÊNCIAS BIBLIOGRÁFICAS}

1. Sanches B, Franco J, Calhau P, Fernandes RM. Antieméticos na gastroenterite aguda em crianças e adolescentes [Antiemetics in acute gastroenteritis in children and adolescents]. Acta Pediatr Port. 2015;46(2):171-4. Portuguese

2. Barreira ER, Magaldi RB. Distonia aguda relacionada ao uso de bromoprida em pacientes pediátricos [Acute dystonia after use of bromopride in pediatric patients]. Rev Paul Pediatr. 2009;27(1):110-4. Portuguese

3. Campbell D. The management of acute dystonic reactions. Aust Prescr. 2001;24(1):19-20.

4. Incecik F, Önlen Y, Özer C, Özcan K. Metoclopramide induced acute dystonic reaction: two case reports. Marmara Med J. 2008;21(2):159-61.

5. Marchetti F, Bonati M, Maestro A, Zanon D, Rovere F, Arrighini A, et al. Oral Ondansetron versus Domperidone for acute gastroenteritis in pediatric emergency departments: multicenter double blind randomized controlled trial. PLoS One. 2016;11(11):e0165441.

\section{CONFLITO DE INTERESSES}

Os autores declaram não ter quaisquer conflitos de interesse.

\section{ENDEREÇO PARA CORRESPONDÊNCIA}

Alexandra Cadete

E-mail: alexandracadete.novo@outlook.com

https://orcid.org/0000-0001-6774-9233

Recebido em 12-03-2017

Aceite para publicação em 08-05-2018

\section{ABSTRACT}

\section{THE IMPORTANCE OF SHARING CLINICAL INFORMATION: CASE REPORT OF AN ADVERSE REACTION}

Introduction: Although not very consensual, antiemetic therapy is widely used in clinical practice in order to minimize dehydration and reduce the need of intravenous fluid therapy. The choice of antiemetic drug is extremely important given the incidence of adverse reactions, which may reach $25 \%$ in pediatric age.

Case description: A 12-year-old young boy was admitted in the emergency department of a private hospital with complaints of headache, fever and an episode of vomiting. Intravenous paracetamol and metoclopramide were administered, with subsequent improvement of the symptoms. However, sometime later, the patient resumed a severe frontal headache and psychomotor agitation, which led to his transfer to the emergency department of the National Health System. At admission, the patient was very anxious, agitated and uncomfortable. At the beginning of the physical examination, he began to exhibit dystonic movements that made communication difficult and rendered the physical examination impossible to perform, but he never lost consciousness. An extrapyramidal reaction to metoclopramide was suspected and intravenous biperiden was administered, with complete reversal of the clinical presentation.

Comment: Although uncommon, acute dystonic reactions may occur within the therapeutic dose of metoclopramide. Family doctors attend patients of all ages; therefore, it is important to keep in mind the possible adverse effects of this medication, as well as to record and transmit information regarding the therapeutic actions performed, which were fundamental in this case. In pediatric age, the administration of others drugs with well-established safety such as ondansetron and domperidone is advised.

Keywords: Extrapyramidal; Metoclopramide; Pediatric; Antiemetics. 